\title{
HOT CARRIER SOLAR CELLS: CHALLENGES AND RECENT PROGRESS
}

\author{
Martin A. Green ${ }^{1}$, Gavin Conibeer ${ }^{1}$, Dirk König ${ }^{1}$, Santosh Shrestha ${ }^{1}$, Shujuan Huang ${ }^{1}$, \\ Pasquale Aliberti ${ }^{1}$, Lara Treiber $^{1}$, Robert Patterson ${ }^{1}$, Binesh Puthen Veettil ${ }^{1}$, Andy Hsieh ${ }^{1}$, Yu Feng $^{1}$, \\ A. Luque ${ }^{2}$, A. Marti ${ }^{2}$, P.G. Linares ${ }^{2}$, E. Cánovas ${ }^{2}$, E. Antolín², D. Fuertes Marrón², C. Tablero ${ }^{2}$, \\ E. Hernández ${ }^{2}$, J-F. Guillemoles ${ }^{3}$, L. Huang ${ }^{3}$, A. Le Bris ${ }^{3}$, T. Schmidt ${ }^{4}$, R. Clady ${ }^{4}$, M. Tayebjee ${ }^{4}$ \\ ${ }^{1}$ ARC Photovoltaics Centre of Excellence, University of New South Wales, Sydney, Australia, 2052 \\ Tel: +61 293854018 Fax: +61 296624240 email: m.green@unsw.edu.au \\ ${ }^{2}$ Instituto de Energia Solar, Universidad Politecnica de Madrid, 28040 Madrid, Spain \\ Tel: +34 915441060 Fax: +34915446341 email: luque@ies-def.upm.es \\ ${ }^{3}$ Institute for Research and Development of Photovoltaic Energy (IRDEP), joint EDSRC-CNRS, Paris \\ Scientifique et de l'Ecole Nationale Supérieure de Chimie de Paris, 6 quai Watier, Chatou, France \\ Tel: +33155426381 Fax: +33144276750 email: if-guillemoles@enscp.fr \\ ${ }^{4}$ School of Chemistry, University of Sydney, Sydney Australia, 2006 \\ Tel: +61 293512781 Fax: +61 293513329 email: t.schmidt@chem.usyd.edu.au
}

\begin{abstract}
The limiting efficiency on the conversion efficiency of terrestrial global sunlight is not circa $31 \%$, as commonly assumed, but $74 \%$. To reach the lowest possible costs and hence to attain its intrinsic potential as a major source of future sustainable energy supplies, it would appear photovoltaics has to evolve to devices targeting the latter efficiency rather than the former. The hot carrier solar cell, although presenting substantial device challenges, is arguably the highest efficiency photovoltaic device concept yet suggested and hence worthy of efforts to investigate its practicality. Challenges in the implementation of hot carrier cells are identified and progress in overcoming these are discussed.
\end{abstract}

\section{INTRODUCTION}

Figure 1 shows the likely cost-efficiency domain of "first generation" wafer-based silicon technology as well as that of "second generation" thin-film cells. First generation devices are presently characterised by manufacturing costs of about US $\$ 200 / \mathrm{m}^{2}$ and module efficiency of about $14 \%\left(140 \mathrm{Wp} / \mathrm{m}^{2}\right)$, giving about $\$ 1.40 / \mathrm{Wp}$ manufacturing costs for large manufacturers [1].

The best thin-film devices on the other hand have manufacturing costs of about $\$ 100 / \mathrm{m}^{2}$ and module efficiency around $10 \%$ (total area) giving manufacturing costs of about $\$ 1.00 / \mathrm{Wp}$ (including overhead [1]). If manufacturing costs similar to thin films can be retained while targeting $74 \%$ efficiency rather than the $31 \%$ value that is the limit for conventional cells, considerable leverage is obtained in reducing the manufacturing cost as shown by the "third generation" region (III) in Fig. 1. Costs/Wp in the $\$ 0.20-\$ 0.50 / \mathrm{Wp}$ range would be feasible with manufacturing costs per unit area similar to those already demonstrated for conventional thin films.

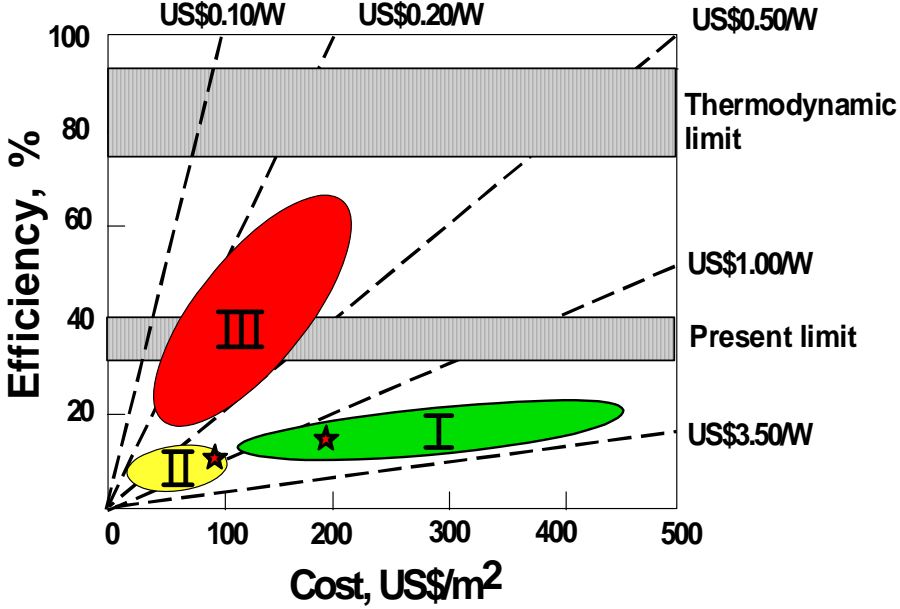

Figure 1 Cost/efficiency regions for three generations of PV technology [2].

Figure 2 shows a range of technologies that are capable of exceeding the conventional PV cell limit. The highest efficiency for a time-symmetric system [2] is obtained for an infinite tandem stack of cells of different bandgap. However, very similarly efficiencies are possible for hot carrier cells, certainly higher in principle than a 6-cell stack that may represent a limit on the number of cells in any practical stack. As such, the hot carrier cell is arguably the highest efficiency solar cell concept yet suggested.

\section{HOT CARRIER CELL PRINCIPLES}

The basic concept underlying hot carrier cell operation is shown in Fig. 3. In a normal cell, a lot of energy is lost through relaxation of photoexcited carriers to energies that 

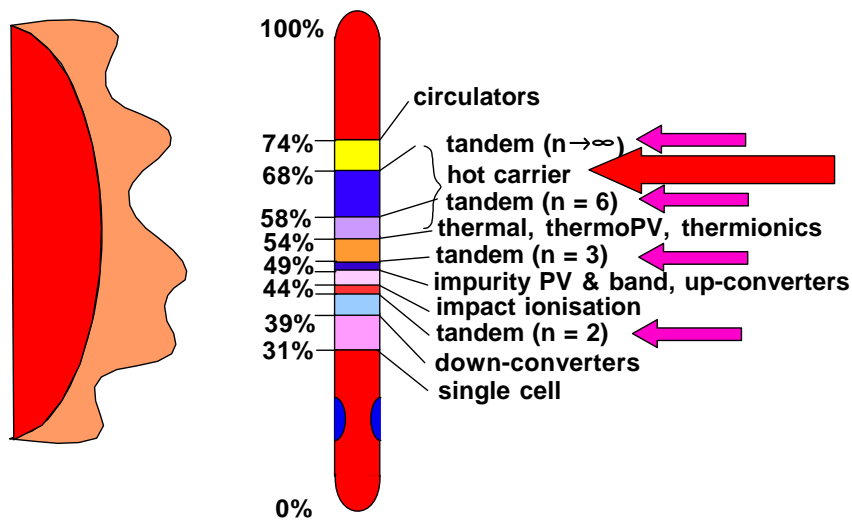

Figure 2 Third generation options [2].

are close to the bandgap (Fig. 3). A high energy blue photon produces the same overall outcome as a lower energy red photon due to this process. In a hot carrier cell, this relaxation is somehow avoided allowing energy in excess of the bandgap to be stored in the photoexcited carrier populations.
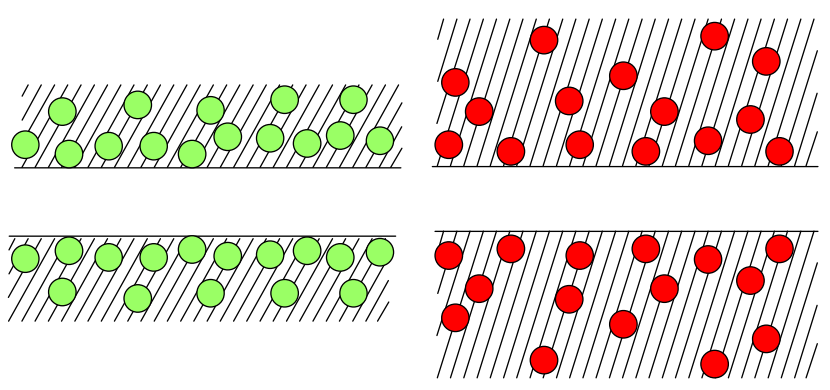

Figure 3 "Cold carrier" (left) and "hot carrier" (right) populations.

The efficiency limits on this approach can be deduced by an extension of the original Shockley-Quiesser approach $[3,4]$ and are shown in Fig. 4. The uppermost curve labeled (1) represents results of an analysis by Ross and Nozik [3] while that labeled (2) represents those from Würfel's analysis [4]. The dashed line shows the conventional "cold carrier limits".

Ross and Nozik's analysis assumes radiative recombination only (zero Auger recombination) whereas Würfel's essentially assumes infinite Auger recombination (not an energy loss process for an ideal hot carrier cell!). If Auger recombination is regarded as an intrinsic material property, the limit would lie between these extremes, suggesting efficiency limits above a 6-cell tandem are possible for material of selected bandgap and Auger recombination properties.

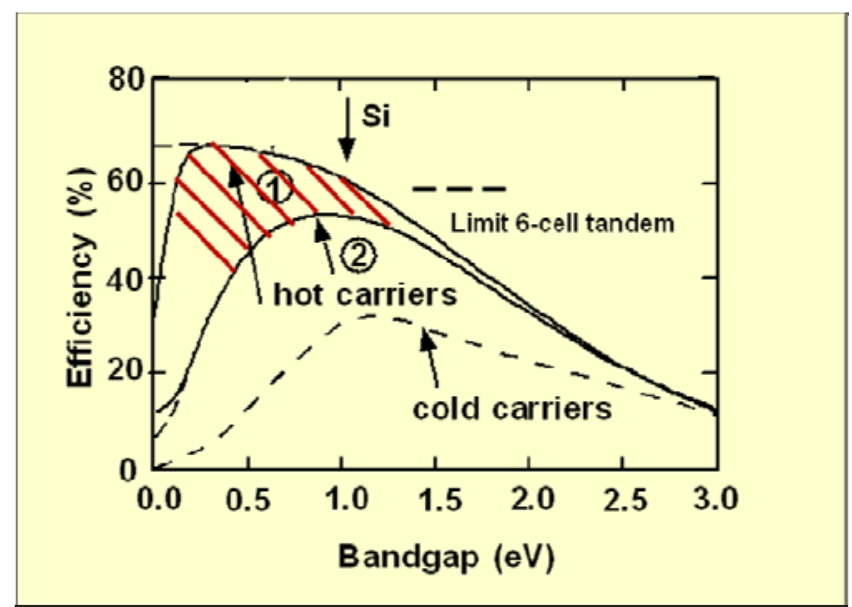

Figure 4 Limiting efficiency versus bandgap for hot and cold carrier devices.

\section{DEVICE CONCEPT}

Figure 5 shows a possible hot carrier device concept. As well as the special absorber properties, special contacts to the outside world are also required.

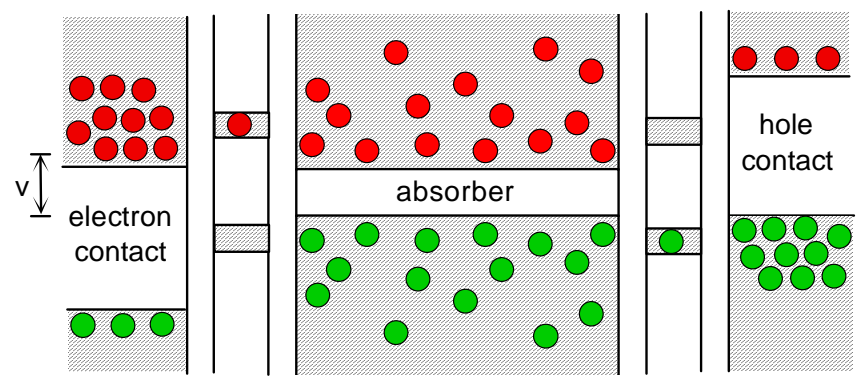

Figure 5 "n-i-p” hot carrier device concept.

In a normal low resistance contact, the nett current flow is the small difference between current flows going in opposite directions. For a hot carrier absorber, the hot carrier flows from the absorber would be almost balanced by cold carrier flows from the contact, destroying the hot carrier effect.

This problem can be avoided if current flow between the absorber and contact occurs over a narrow range of energies.

Resonant tunneling through a single layer of quantum dots in a dielectric matrix is one way of achieving this (Fig. 6) [2]. An additional refinement shown in Fig. 5 is to use large bandgap, heavily doped semiconductors as the contact [5]. This not only closes off unwanted transport channels as suggested by Fig. 5, but also provides the "built-in" potential against which the photovoltage can operate. 


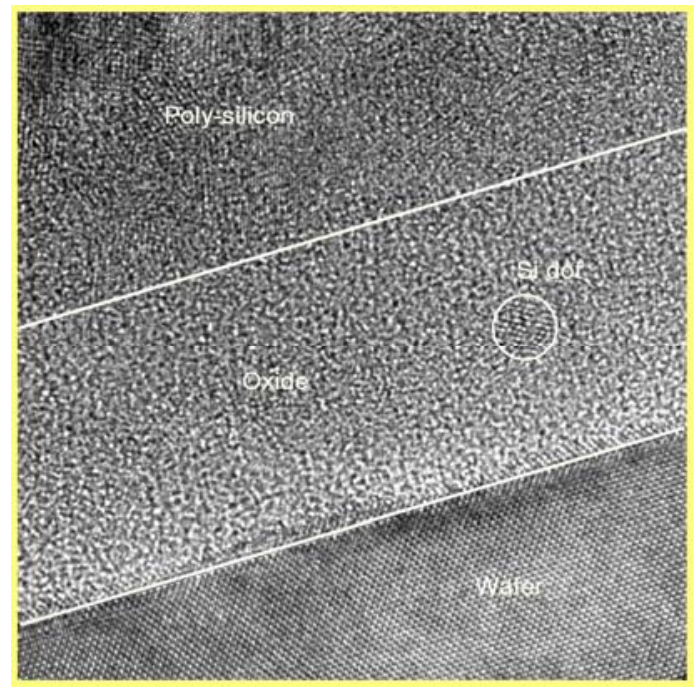

Figure 6 Resonant quantum-dot structure (Si dots between two bulk Si layers).

\section{HOT PHONONS}

The most promising way of preventing the energy relaxation of photoexcited electrons may be to create a hot phonon population by making it difficult for optical phonons to decompose into acoustic phonons

By way of example, Fig. 7 shows the energy-crystal momentum relationship and phonon density of states (DOS) calculated for InN [6]. A clear phononic bandgap exists between the low energy acoustic phonons (frequencies below about $200 / \mathrm{cm}$ ) and the high energy optical phonons (frequencies above circa 450/cm).

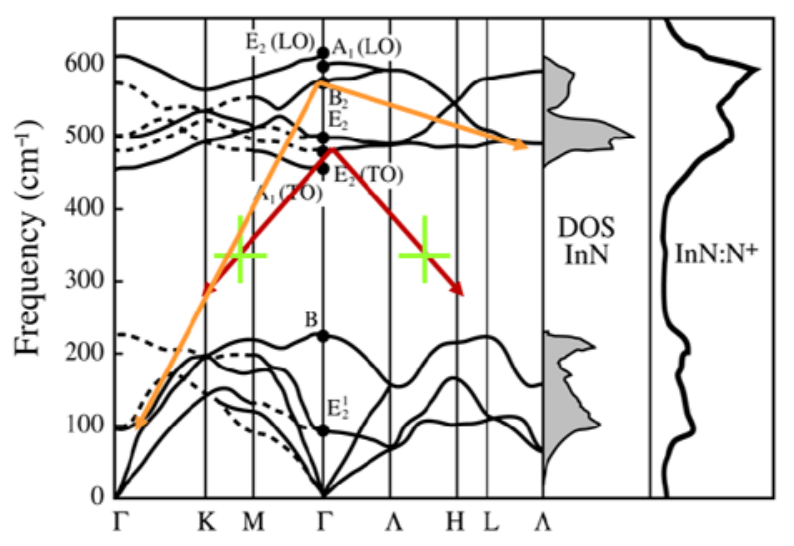

Figure 7 Phononic properties of $\operatorname{InN}[6]$.

In fact, for the highest energy acoustic phonons, the light $\mathrm{N}$ atoms are stationary and the heavy In atoms oscillate against them with an associated energy proportional to the inverse of the In atom mass.

Conversely, for the lowest energy optical phonons, the In atoms are stationary and the light $\mathrm{N}$ atoms vibrate vigorously against them. The difference in the associated energy explains the gap. Moreover, since In atoms are more than 4 times heavier than $\mathrm{N}$ atoms, the gap is larger than the highest energy in the acoustic band.

The way a photoexcited carrier normally relaxes to the band edge is by emitting high energy optical phonons. These have relatively low group velocity (as determined by the flatness of the optical bands) and so are relatively immobile. They can also be trapped into the absorber by appropriate boundary layers.

However, an optical phonon can decay normally into 2 acoustic phonons in a process that conserves both energy and momentum (Klemens process).

Acoustic phonons are relatively mobile and more difficult to trap in the absorber and so can carry off the energy. However, in InN, the Klemens mechanism is not possible since the optical phonon energy is more than twice that of any acoustic phonon. Optical phonons must decay into at least 3 acoustic phonons, suppressing delay rates, with some experimental evidence for this effect available [7].

This gives real prospects for the optical phonons population to be higher than in thermal equilibrium (ie. to become hot). The situation is analogous to that of a semiconductor with its 2 bands when electrons are photoexcited. A hot optical phonon population in turn slows the rate of electrons can impart energy to the optical phonons, slowing their cooling.

Although $\operatorname{InN}$ has a near-ideal bandgap for a hot-carrier cell, In is a scarce element thereby making the material unattractive as a third-generation option. Other light-heavy element combinations may be more interesting. Alternatively, similar phononic bandgaps may be able to be created in nano-engineered materials.

\section{POSSIBLE DEVICE IMPLEMENTAION}

Based on the above discussion, Fig. 8 shows a possible hot carrier cell concept. An n-type transparent conducting oxide is used as the top contact, with a heavily doped $p$ type contact at the rear (transparency might be an advantage in light-trapping).

Small quantum dots with high resonant state energy are used to provide resonant electron and hole transport paths between the absorber and the respective contacts. The absorber itself is made of larger, uniformly spaced, coreshell quantum dots.

Our studies suggest that having three mechanical properties to work with, namely the stiffness of the core, of 
the shell and of the matrix material, provides the flexibility required to engineer the desired phononic gaps.

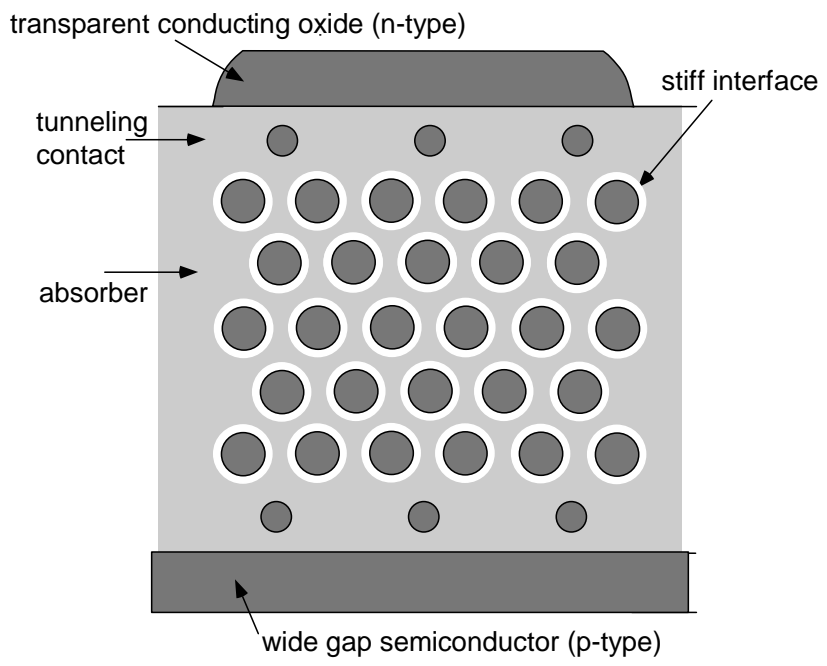

Figure 8 Conceptual hot carrier cell based on nanoengineered phononic properties.

\section{CONCLUSIONS}

Hot carrier cells are arguably the highest efficiency photovoltaic concept yet suggested. Unusual monoenergetic contacts and special absorber properties are required. The selective energy contacts appear achievable using resonant tunneling transport. Phononic engineering appears the best option for slowing carrier cooling in the absorber although good new ideas here are needed

\section{REFERENCES}

[1] J.Song, R. Boas, C. Bolman, M. Meyers, M. Rogol and R. Trangucci, "The True Cost of Solar Power: How Low Can You Go?" Photon Consulting, April 2010.

[2] M.A. Green, "Third Generation Photovoltaics: Advanced Solar Electricity Generation", Spriinger-Verlag, Berlin, 2003.

[3] R.T. Ross and A.J. Nozik, "Efficiency of hot-carrier solar energy converters", Journal of Applied Physics 53, p. $3813,1982$.

[4] P. Würfel, "Solar energy conversion with hot electrons from impact ionisation", Solar Energy Materials and Solar Cells 46, pp. 43-52, 1997.

[5] D. König, K. Casalenuovo, Y. Takeda, G. Conibeer, J.F. Guillemoles, R. Patterson, L.M. Huang, M.A. Green, "Hot Carrier Solar Cells: Principles, Design and Materials", Physica E: Low-dimensional Systems and Nanostructures,
In Press corrected proof available online Jan 2010, DOI 10.1016/j.physe.2009.12.032.

[6] V. Yu. Davydov, V. V. Emtsev, I. N. Goncharuk, A. N. Smirnov, V. D. Petrikov, V. V. Mamutin, V. A. Vekshin, S. V. Ivanov, M. B. Smirnov, and T. Inushima, "Experimental and theoretical studies of phonons in hexagonal InN", Applied Physics Letter 75, pp. 3297-3300, 1999.

[7] J.W. Pomeroy, M. Kuball, H. Lu, W.J. Schaff, X. Wang and A. Yoshikawa, "Phonon lifetimes and phonon decay in InN", Applied Physics Letters 86, pp. 223501-3, 2005. 